\title{
A subdivision-based approach for singularly perturbed boundary value problem
}

\author{
Ghulam Mustafa', Syeda Tehmina Ejaz ${ }^{1,2}$, Dumitru Baleanu ${ }^{3,4,5}$, Abdul Ghaffar ${ }^{6,7}$ and \\ Kottakkaran Sooppy Nisar ${ }^{8 *}$ (i)
}

\section{"Correspondence: \\ n.sooppy@psau.edu.sa \\ ${ }^{8}$ Department of Mathematics, College of Arts and Sciences, Prince Sattam bin Abdulaziz University, Wadi Aldawaser, Saudi Arabia Full list of author information is available at the end of the article}

\begin{abstract}
A numerical approach for solving second order singularly perturbed boundary value problems (SPBVPs) is introduced in this paper. This approach is based on the basis function of a 6-point interpolatory subdivision scheme. The numerical results along with the convergence, comparison and error estimation of the proposed approach are also presented.
\end{abstract}

MSC: Primary 65Nxx; 65L11; 65L10; 65L99; 65L60; secondary 65D07

Keywords: Singularly perturbed; Boundary value problem; Ordinary differential equation; Interpolating schemes; Subdivision scheme

\section{Introduction}

The singularly perturbed boundary value problems (SPBVPs) frequently occur in the different areas of physical phenomena. Specifically, these occur in the fields of fluid dynamic, elasticity, neurobiology, quantum mechanics, oceanography, and reactor diffusion process. These problems often have sharp boundary layers. These boundary layers usually appear as a multiple of the highest derivative. Their small values cause trouble in different numerical schemes for the solution of SPBVPs. Therefore, it is important to find the numerical and analytic solutions of these types of problems. The different second order SPBVPs have different expressions but we deal with the following:

$$
\begin{aligned}
& \varepsilon Z^{\bullet \bullet}(u)=p(u) Z^{\bullet}(u)+q(u) Z(u)+g(u), \\
& Z(a)=\alpha_{0}, \quad Z(b)=\alpha_{1}, \quad a \leq u \leq b,
\end{aligned}
$$

where $0<\varepsilon<1$, while $p(u), q(u), g(u)$ are bounded and real valued functions. $g(u), \alpha_{0}, \alpha_{1}$ depends on $\varepsilon$. We may refer to Ascher et al. [1] for more details as regards such a type of SPBVPs.

Here, we first present short review of different methods for the solution of second order SPBVPs; then we discuss a subdivision-based solution of SPBVPs.

The second order SPBVPs were solved based on cubic spline scheme by Aziz and Khan $[2,6]$ in 2005. These problems were also solved by Bawa and Natesan [3] in the same year.

(c) The Author(s) 2020. This article is licensed under a Creative Commons Attribution 4.0 International License, which permits use, sharing, adaptation, distribution and reproduction in any medium or format, as long as you give appropriate credit to the original author(s) and the source, provide a link to the Creative Commons licence, and indicate if changes were made. The images or other third party material in this article are included in the article's Creative Commons licence, unless indicated otherwise in a credit line to the material. If material is not included in the article's Creative Commons licence and your intended use is not permitted by statutory regulation or exceeds the permitted use, you will need to obtain permission directly from the copyright holder. To view a copy of this licence, visit http://creativecommons.org/licenses/by/4.0/. 
They have used quintic spline based approximating schemes. Kadalbajoo and Aggarwal [5] and Tirmizi et al. [17] solved self-adjoint SPBVPs by using B-spline collocation and nonpolynomial spline function schemes, respectively. Kumar and Mehra [7] and Pandit and Kumar [14] solved SPBVPs by a wavelet optimized difference and uniform Haar wavelet methods, respectively.

The second order SPBVPs were also solved by $[9,12,13]$. They have used finite difference scheme for the solution.

The linear SPBVPs were solved by $[4,11,15]$. They have used interpolating subdivision schemes for this purpose. The solution of second order SPBVPs by subdivision techniques did not reported yet. We develop an algorithm by using a 6-point interpolating subdivision scheme (6PISS) [8]. We have

$$
\left\{\begin{array}{l}
Q_{2 i}^{k+1}=Q_{i}^{k}, \\
Q_{2 i+1}^{k+1}=\mu\left(Q_{i-2}^{k}+Q_{i+3}^{k}\right)-\left(3 \mu+\frac{1}{16}\right)\left(Q_{i-1}^{k}+Q_{i+2}^{k}\right)+\left(2 \mu+\frac{9}{16}\right)\left(Q_{i}^{k}+Q_{i+1}^{k}\right),
\end{array}\right.
$$

where the scheme is $C^{2}$-continuous for $0<\mu<0.042$. It has support width $(-5,5)$. It has fourth order of approximation. It satisfies the 2 -scale relation

$$
\begin{aligned}
\rho(u)= & \rho(2 u)+\left[\mu\{\rho(2 u-1)+\rho(2 u+1)\}-\left(3 \mu+\frac{1}{16}\right)\{\rho(2 u-3)+\rho(2 u\right. \\
& \left.+3)\}+\left(2 \mu+\frac{9}{16}\right)\{\rho(2 u-5)+\rho(2 u+5)\}\right], \quad u \in \mathbb{R},
\end{aligned}
$$

where

$$
\rho(u)= \begin{cases}1 & \text { for } u=0 \\ 0 & \text { for } u \neq 0 .\end{cases}
$$

Here is the layout of the rest of the work. In Sect. 2, we first find the derivatives of $\rho(u)$ then by using them we develop the collocation algorithm. The convergence of the method is discussed in Sect. 3. In Sect. 4, we present the numerical solutions of different problems. The comparison of the solutions obtained by different methods is also offered. Section 5 deals with our conclusion.

\section{The numerical algorithm}

In this section, we develop an algorithm to deal with second order SPBVPs. First we discuss the derivatives of 2 -scale relations known as basis functions of the subdivision scheme.

\subsection{Derivatives of 2-scale relations}

The 6PISS is $C^{2}$-continuous by [8], so its 2-scale relations $\rho(u)$ are also $C^{2}$-continuous. First we find the eigenvectors (both left and right) of the subdivision matrix of 6PISS then we find the derivatives of $\rho(u)$. For simplicity, we choose $\mu=0.04$ to find eigenvectors. We use a similar approach to $[4,11]$ to find the derivatives. The first two derivatives of the 2-scale relation are given in Table 1. 
Table 1 2-scale relation and its derivatives

\begin{tabular}{llllll}
\hline & $i$ & & & & \\
\cline { 2 - 6 } & 0 & \pm 1 & \pm 2 & \pm 3 & \pm 4 \\
\hline$\rho(i)$ & 1 & 0 & 0 & 0 & 0 \\
$\rho^{\bullet}(i)$ & 0 & $\pm \frac{9100}{4313}$ & $\mp \frac{19,673}{25,878}$ & $\pm \frac{1600}{12,939}$ & $\pm \frac{128}{12,939}$ \\
$\rho^{\bullet \bullet}(i)$ & $-\frac{14,431}{4224}$ & $\frac{4325}{2112}$ & $-\frac{1575}{2816}$ & $\frac{25}{132}$ & $\frac{1}{33}$ \\
\hline
\end{tabular}

\subsection{The 6PISS based algorithm}

Let $m$ be the indexing parameter which might be equal to or greater than the last right end integral value of the right eigenvector corresponding to the eigenvalue $\frac{1}{2}$ of the subdivision matrix for (3). Some useful notations depending on the indexing parameter $m$ are defined as $h=1 / m$ and $v_{\kappa_{1}}=\kappa_{1} / m=i h$ with $\kappa_{1}=0,1,2, \ldots, m$. Finally, we suppose that the approximate solution of (1) is

$$
D(v)=\sum_{\kappa_{1}=-4}^{m+4} d_{\kappa_{1}} \rho\left(\frac{v-v_{\kappa_{1}}}{h}\right), \quad 0 \leq v \leq 1
$$

where $\left\{d_{\kappa_{1}}\right\}$ are the unknowns to be determined; then

$$
a D^{\bullet \bullet}\left(v_{\kappa}\right)=p\left(v_{\kappa}\right) D^{\bullet}\left(v_{\kappa}\right)+q\left(v_{\kappa}\right) D\left(v_{\kappa}\right)+g\left(v_{\kappa}\right), \quad \kappa=0,1,2, \ldots, m,
$$

with given boundary conditions at both ends of the interval

$$
D(0)=\alpha_{0}, \quad D(1)=\alpha_{1} .
$$

From (6), we have

$$
\begin{aligned}
& D^{\bullet}\left(v_{\kappa}\right)=\frac{1}{h} \sum_{\kappa_{1}=-4}^{m+4} d_{\kappa_{1}} \rho \cdot\left(\frac{v_{\kappa}-v_{\kappa_{1}}}{h}\right), \\
& D^{\bullet \bullet}\left(v_{\kappa}\right)=\frac{1}{h^{2}} \sum_{\kappa_{1}=-4}^{m+4} d_{\kappa_{1}} \rho \bullet\left(\frac{v_{\kappa}-v_{\kappa_{1}}}{h}\right) .
\end{aligned}
$$

We get the system of equations by using (6) and (8) in (7),

$$
\begin{gathered}
a \sum_{\kappa_{1}=-4}^{m+4} d_{\kappa_{1}} \rho \cdot \bullet\left(\frac{v_{\kappa}-v_{\kappa_{1}}}{h}\right)-h p_{\kappa} \sum_{\kappa_{1}=-4}^{m+4} d_{\kappa_{1}} \rho \cdot\left(\frac{v_{\kappa}-v_{\kappa_{1}}}{h}\right) \\
-h^{2} q_{\kappa} \sum_{\kappa_{1}=-4}^{m+4} d_{\kappa_{1}} \rho\left(\frac{v_{\kappa}-v_{\kappa_{1}}}{h}\right)=h^{2} g_{\kappa},
\end{gathered}
$$

where $A_{\kappa}=p\left(v_{\kappa}\right), q_{\kappa}=q\left(v_{\kappa}\right)$ and $g_{\kappa}=g\left(v_{\kappa}\right)$. This implies

$$
\sum_{\kappa_{1}=-4}^{m+4} d_{\kappa_{1}}\left\{a \rho \cdot\left(\frac{v_{\kappa}-v_{\kappa_{1}}}{h}\right)-h p_{\kappa} \rho \cdot\left(\frac{v_{\kappa}-v_{\kappa_{1}}}{h}\right)-h^{2} q_{\kappa} \rho\left(\frac{v_{\kappa}-v_{\kappa_{1}}}{h}\right)\right\}=h^{2} g_{\kappa} .
$$


This further implies

$$
\sum_{\kappa_{1}=-4}^{m+4} d_{\kappa_{1}}\left\{a \rho^{\bullet \bullet}\left(\kappa-\kappa_{1}\right)-h p_{\kappa} \rho^{\bullet}\left(\kappa-\kappa_{1}\right)-h^{2} q_{\kappa} \rho\left(\kappa-\kappa_{1}\right)\right\}=h^{2} g_{\kappa},
$$

where $\kappa=0,1,2, \ldots, m$ and $v_{\kappa_{1}}=i h$ or $v_{\kappa}=j h$. By using the notation " $\rho\left(\kappa_{1}\right)=\rho_{\kappa_{1}}$ ", (9) can be written as

$$
\sum_{\kappa_{1}=-4}^{m+4} d_{\kappa_{1}}\left\{a \rho_{\kappa-\kappa_{1}}^{\bullet \bullet}-h p_{\kappa} \rho_{\kappa-\kappa_{1}}^{\bullet}-h^{2} q_{\kappa} \rho_{\kappa-\kappa_{1}}\right\}=h^{2} g_{\kappa}, \quad \kappa=0,1,2, \ldots, m
$$

As we observe from Table $1, \rho_{-\kappa_{1}}^{\bullet}=-\rho_{\kappa_{1}}^{\bullet}$ and $\rho_{-\kappa_{1}}^{\bullet \bullet}=\rho_{\kappa_{1}}^{\bullet \bullet}$, for $\kappa=0,1,2, \ldots, m,(10)$ becomes

$$
\sum_{\kappa_{1}=-4}^{m+4} d_{\kappa_{1}}\left\{a \rho_{\kappa_{1}-\kappa}^{\bullet \bullet}+h p_{\kappa} \rho_{\kappa_{1}-\kappa}^{\bullet}-h^{2} q_{\kappa} \rho_{\kappa_{1}-\kappa}\right\}=h^{2} g_{\kappa}
$$

The above system of equations is summarized in the following proposition.

Proposition 1 The equivalent form of the system (11) is

$$
\sum_{\kappa_{1}=-4}^{4} d_{\kappa+\kappa_{1}} \tau_{\kappa_{1}}^{\kappa}=h^{2} g_{\kappa}, \quad \kappa=0,1,2, \ldots, m
$$

where

$$
\tau_{\kappa_{1}}^{\kappa}= \begin{cases}a \rho_{0}^{\bullet \bullet}-h^{2} q_{\kappa}, & \text { for } \kappa_{1}=0, \\ a \rho_{\kappa_{1}}^{\bullet \bullet}-h p_{\kappa} \rho_{\kappa_{1}}^{\bullet}, & \text { for } \kappa_{1} \neq 0 .\end{cases}
$$

Proof Substituting $\kappa=0$ in (11), we get

$$
\sum_{\kappa_{1}=-4}^{m+4} d_{\kappa_{1}}\left\{a \rho_{\kappa_{1}}^{\bullet \bullet}+h A_{0} \rho_{\kappa_{1}}^{\bullet}-h^{2} q_{0} \rho_{\kappa_{1}}\right\}=h^{2} g_{0}, \quad \kappa=0,1,2, \ldots, m .
$$

By expanding the above equation, we get

$$
\begin{aligned}
& d_{-4}\left\{a \rho_{-4}^{\bullet \bullet}+h p_{0} \rho_{-4}^{\bullet}-h^{2} q_{0} \rho_{-4}\right\}+d_{-3}\left\{a \rho_{-3}^{\bullet \bullet}+h p_{0} \rho_{-3}^{\bullet}-h^{2} q_{0} \rho_{-3}\right\}+\cdots \\
& \quad+d_{0}\left\{a \rho_{0}^{\bullet \bullet}+h p_{0} \rho_{0}^{\bullet}-h^{2} q_{0} \rho_{0}\right\}+\cdots+d_{m+3}\left\{a \rho_{m+3}^{\bullet \bullet}+h p_{0} \rho_{m+3}^{\bullet}-h^{2} q_{0} \rho_{m+3}\right\} \\
& +d_{m+4}\left\{a \rho_{m+4}^{\bullet \bullet}+h p_{0} \rho_{m+4}^{\bullet}-h^{2} q_{0} \rho_{m+4}\right\}=h^{2} g_{0} .
\end{aligned}
$$

Since the support width of the 6PISS is $(-5,5)$ therefore the graphs of $\rho_{\kappa_{1}}^{\bullet}$ and $\rho_{\kappa_{1}}^{\bullet \bullet}$ cannot be zero over the domain $[-4,4]$ but their graphs away from it will be zero. This simplifies as

$$
\begin{aligned}
& d_{-4}\left\{a \rho_{-4}^{\bullet \bullet}+h p_{0} \rho_{-4}^{\bullet}-h^{2} q_{0} \rho_{-4}\right\}+d_{-3}\left\{a \rho_{-3}^{\bullet \bullet}+h p_{0} \rho_{-3}^{\bullet}-h^{2} q_{0} \rho_{-3}\right\}+d_{-2}\left\{a \rho_{-2}^{\bullet \bullet}\right. \\
& \left.+h p_{0} \rho_{-2}^{\bullet}-h^{2} q_{0} \rho_{-2}\right\}+d_{-1}\left\{a \rho_{-1}^{\bullet \bullet}+h p_{0} \rho_{-1}^{\bullet}-h^{2} q_{0} \rho_{-1}\right\}+d_{0}\left\{a \rho_{0}^{\bullet \bullet}+h p_{0} \rho_{0}^{\bullet}\right.
\end{aligned}
$$




$$
\begin{aligned}
& \left.-h^{2} q_{0} \rho_{0}\right\}+d_{1}\left\{a \rho_{1}^{\bullet \bullet}+h p_{0} \rho_{1}^{\bullet}-h^{2} q_{0} \rho_{1}\right\}+d_{2}\left\{a \rho_{2}^{\bullet \bullet}+h p_{0} \rho_{2}^{\bullet}-h^{2} q_{0} \rho_{2}\right\}+d_{3}\left\{a \rho_{3}^{\bullet \bullet}\right. \\
& \left.+h p_{0} \rho_{3}^{\bullet}-h^{2} q_{0} \rho_{3}\right\}+d_{4}\left\{a \rho_{4}^{\bullet \bullet}+h p_{0} \rho_{4}^{\bullet}-h^{2} q_{0} \rho_{4}\right\}=h^{2} g_{0} .
\end{aligned}
$$

By substituting the values of $\rho_{\kappa_{1}}$ and $\rho_{0}^{\bullet}=0$, we get

$$
\begin{aligned}
& d_{-4}\left\{a \rho_{-4}^{\bullet \bullet}+h p_{0} \rho_{-4}^{\bullet}\right\}+d_{-3}\left\{a \rho_{-3}^{\bullet \bullet}+h p_{0} \rho_{-3}^{\bullet}\right\}+d_{-2}\left\{a \rho_{-2}^{\bullet \bullet}+h p_{0} \rho_{-2}^{\bullet}\right\} \\
& +d_{-1}\left\{a \rho_{-1}^{\bullet \bullet}+h p_{0} \rho_{-1}^{\bullet}\right\}+d_{0}\left\{a \rho_{0}^{\bullet \bullet}-h^{2} q_{0} \rho_{0}\right\}+d_{1}\left\{a \rho_{1}^{\bullet \bullet}+h p_{0} \rho_{1}^{\bullet}\right\} \\
& +d_{2}\left\{a \rho_{2}^{\bullet \bullet}+h p_{0} \rho_{2}^{\bullet}\right\}+d_{3}\left\{a \rho_{3}^{\bullet \bullet}+h p_{0} \rho_{3}^{\bullet}\right\}+d_{4}\left\{a \rho_{4}^{\bullet \bullet}+h p_{0} \rho_{4}^{\bullet}\right\}=h^{2} g_{0} .
\end{aligned}
$$

If

$$
\begin{aligned}
& \tau_{ \pm 4}^{0}=a \rho_{ \pm 4}^{\bullet \bullet}+h p_{0} \rho_{ \pm 4}^{\bullet}, \quad \tau_{ \pm 3}^{0}=a \rho_{ \pm 3}^{\bullet \bullet}+h p_{0} \rho_{ \pm 3}^{\bullet}, \quad \tau_{ \pm 2}^{0}=a \rho_{ \pm 2}^{\bullet \bullet}+h p_{0} \rho_{ \pm 2}^{\bullet}, \\
& \tau_{ \pm 1}^{0}=a \rho_{ \pm 1}^{\bullet \bullet}+h p_{0} \rho_{ \pm 1}^{\bullet}, \quad \tau_{0}^{0}=a \rho_{0}^{\bullet \bullet}-h^{2} q_{0},
\end{aligned}
$$

the above equation becomes

$$
\sum_{\kappa_{1}=-4}^{4} d_{\kappa_{1}} \tau_{\kappa_{1}}^{0}=h^{2} g_{0}
$$

Similarly, for $\kappa=1,2,3, \ldots, m$, we get

$$
\sum_{\kappa_{1}=-4}^{4} d_{\kappa_{1}+\kappa} \tau_{\kappa_{1}}^{\kappa}=h^{2} g_{\kappa}
$$

where, for $\kappa_{1}=-4,-3, \ldots, 3,4$ and $\kappa=1,2,3, \ldots, m$, we have

$$
\tau_{\kappa_{1}}^{\kappa}= \begin{cases}a \rho_{0}^{\bullet \bullet}-h^{2} q_{\kappa} & \text { for } \kappa_{1}=0 \\ a \rho_{\kappa_{1}}^{\bullet \bullet}-h p_{\kappa} \rho_{\kappa_{1}}^{\bullet} & \text { for } \kappa_{1} \neq 0\end{cases}
$$

The proof has been completed.

\subsubsection{Matrix representation of the linear system}

The matrix representation of the linear system (12) is given by

$$
\mathbb{S D}=\mathbb{G}_{1}
$$

where

$$
\mathbb{S}=\left(\xi_{s}^{r-1}\right)_{(m+1) \times(m+9)}
$$

where $r=1,2, \ldots, m+2$ and $s=-4,-3, \ldots, m+3, m+4$ represent row and column, respectively, and

$$
\xi_{s}^{r-1}= \begin{cases}\tau_{\kappa_{1}}^{r-1}, & \text { for }-4 \leq \kappa_{1} \leq 4 \\ 0, & \text { otherwise }\end{cases}
$$


The column matrices $\mathbb{D}$ and $\mathbb{G}_{1}$ are given by

$$
\mathbb{D}=\left(d_{-4}, d_{-3}, \ldots, d_{m+3}, d_{m+4}\right)^{T}
$$

and

$$
\mathbb{G}_{1}=h^{2} \times\left(g_{0}, g_{1}, \ldots, g_{m-1}, g_{m}\right)^{T} .
$$

The system (14) in its present form does not have a unique solution. We need eight extra equations to get its unique solution. Luckily, two equations can be obtained from (2), i.e., $D(0)$ and $D(1)$ and for the remaining six equations, we move to the next section.

\subsubsection{End point constraints}

If the data points are given then the 6PISS is suitable to fit the data with a fourth order of approximation. So we use the fourth order polynomials to define the constraints at the end points. Here we suggest two types of polynomials i.e. the simple cubic polynomial (i.e. a polynomial of order 4) and cardinal basis function-based cubic polynomial, to get the constraints.

C-1: Constraints by polynomial of degree three: We use the fourth order polynomial $C_{1}(v)$ which interpolates the data $\left(v_{\kappa_{1}}, d_{\kappa_{1}}\right)$ for $0 \leq \kappa_{1} \leq 3$ to compute the left end points $d_{-3}, d_{-2}, d_{-1}$. Precisely, we have

$$
d_{-\kappa_{1}}=C_{1}\left(-v_{\kappa_{1}}\right), \quad \kappa_{1}=1,2,3,
$$

where

$$
C_{1}\left(v_{\kappa_{1}}\right)=\sum_{\kappa=1}^{4}\left(\begin{array}{l}
4 \\
\kappa
\end{array}\right)(-1)^{\kappa+1} D\left(v_{\kappa_{1}-\kappa}\right) .
$$

Since by (6), $D\left(v_{\kappa_{1}}\right)=d_{\kappa_{1}}$ for $\kappa_{1}=1,2,3$ then, by replacing $v_{\kappa_{1}}$ by $-v_{\kappa_{1}}$, we have

$$
C_{1}\left(-v_{\kappa_{1}}\right)=\sum_{\kappa=1}^{4}\left(\begin{array}{l}
4 \\
\kappa
\end{array}\right)(-1)^{\kappa+1} d_{-\kappa_{1}+\kappa}
$$

Hence, we get the following three constraints defined at the left end points:

$$
\sum_{\kappa=0}^{4}\left(\begin{array}{l}
4 \\
\kappa
\end{array}\right)(-1)^{\kappa} d_{-\kappa_{1}+\kappa}=0, \quad \kappa_{1}=1,2,3
$$

A similar procedure is adopted for the right end i.e. we can define $d_{\kappa_{1}}=C_{1}\left(v_{\kappa_{1}}\right), \kappa_{1}=$ $m+1, m+2, m+3$ and

$$
C_{1}\left(v_{\kappa_{1}}\right)=\sum_{\kappa=1}^{4}\left(\begin{array}{l}
4 \\
\kappa
\end{array}\right)(-1)^{\kappa+1} d_{\kappa_{1}-\kappa} .
$$


So the following three constraints are defined at the right end:

$$
\sum_{\kappa=0}^{4}\left(\begin{array}{l}
4 \\
\kappa
\end{array}\right)(-1)^{\kappa} d_{\kappa_{1}-\kappa}=0, \quad \kappa_{1}=m+1, m+2, m+3
$$

C-2: Constraints by cardinal basis functions: The following fourth order polynomial $C_{2}(v)$ can be used to find the left points $d_{-3}, d_{-2}, d_{-1}$ :

$$
d_{-\kappa_{1}}=C_{2}\left(-v_{\kappa_{1}}\right), \quad \kappa_{1}=1,2,3,
$$

where

$$
C_{2}(v)=d_{0} \zeta_{0}\left(\frac{v-v_{0}}{h}\right)+d_{1} \zeta_{1}\left(\frac{v-v_{0}}{h}\right)+d_{0}^{\bullet \bullet} \zeta_{0}^{*}\left(\frac{v-v_{0}}{h}\right)+d_{1}^{\bullet \bullet} \zeta_{1}^{*}\left(\frac{v-v_{0}}{h}\right),
$$

while the basis functions are given by

$$
\begin{aligned}
& \zeta_{0}\left(\frac{v-v_{0}}{h}\right)=1-\left(\frac{v-v_{0}}{h}\right), \\
& \zeta_{1}\left(\frac{v-v_{0}}{h}\right)=\left(\frac{v-v_{0}}{h}\right), \\
& \zeta_{0}^{*}\left(\frac{v-v_{0}}{h}\right)=-\frac{1}{6}\left(\frac{v-v_{0}}{h}\right)\left(\frac{v-v_{0}}{h}-1\right)\left(\frac{v-v_{0}}{h}-2\right), \\
& \zeta_{1}^{*}\left(\frac{v-v_{0}}{h}\right)=\frac{1}{6}\left(\frac{v-v_{0}}{h}\right)\left(\frac{v-v_{0}}{h}-1\right)\left(\frac{v-v_{0}}{h}+1\right),
\end{aligned}
$$

and for $t=0,1$

$$
d_{t}^{\bullet \bullet}=p\left(\frac{v_{t}-v_{0}}{h}\right) d_{t}+q\left(\frac{v_{t}-v_{0}}{h}\right) d_{t}+g\left(\frac{v_{t}-v_{0}}{h}\right) .
$$

A similar procedure is adopted for the right end points $d_{\kappa_{1}}=C_{2}\left(-v_{\kappa_{1}}\right), \kappa_{1}=m+1, m+$ $2, m+3$, where

$$
\begin{aligned}
C_{2}(v)= & d_{m} \zeta_{m}\left(\frac{v-v_{m}}{h}\right)+d_{m+1} \zeta_{m+1}\left(\frac{v-v_{0}}{h}\right)+d_{m}^{\bullet \bullet} \zeta_{m}^{*}\left(\frac{v-v_{0}}{h}\right) \\
& +d_{m+1}^{\bullet \bullet} \zeta_{m+1}^{*}\left(\frac{v-v_{0}}{h}\right)
\end{aligned}
$$

and

$$
\begin{aligned}
& \zeta_{m}\left(\frac{v-v_{m}}{h}\right)=1-\left(\frac{v-v_{m}}{h}\right), \\
& \zeta_{m+1}\left(\frac{v-v_{m}}{h}\right)=\left(\frac{v-v_{m}}{h}\right), \\
& \zeta_{m}^{*}\left(\frac{v-v_{m}}{h}\right)=-\frac{1}{6}\left(\frac{v-v_{m}}{h}\right)\left(\frac{v-v_{m}}{h}-1\right)\left(\frac{v-v_{m}}{h}-2\right), \\
& \zeta_{m+1}^{*}\left(\frac{v-v_{m}}{h}\right)=\frac{1}{6}\left(\frac{v-v_{m}}{h}\right)\left(\frac{v-v_{m}}{h}-1\right)\left(\frac{v-v_{m}}{h}+1\right),
\end{aligned}
$$


and for $t=m, m+1$

$$
d_{t}^{\bullet \bullet}=p\left(\frac{v_{t}-v_{m}}{h}\right) d_{t}+q\left(\frac{v_{t}-v_{m}}{h}\right) d_{t}+g\left(\frac{v_{t}-v_{m}}{h}\right) .
$$

\subsubsection{Stable singularly perturbed system}

Finally, we get a stable singularly perturbed system with $m+9$ unknowns and $m+9$ equations obtained from (2), (12), (18) and (19) or (20) and (21).

By C-1 constrants: If we use (2), (12), (18) and (19) then the system can expressed as

$$
\mathbb{S}_{1} \mathbb{D}=\mathbb{G}
$$

where $\mathbb{S}_{1}=\left(\mathbb{S}_{L_{1}}^{T}, \mathbb{S}^{T}, \mathbb{S}_{R_{1}}^{T}\right)^{T}, \mathbb{S}$ is defined by (15). The matrix $\left[\mathbb{S}_{L_{1}}\right]_{4 \times(m+9)}$ is defined as

$$
\mathbb{S}_{L_{1}}=\left(\begin{array}{ccccccccccccc}
0 & 1 & -4 & 6 & -4 & 1 & 1 & 0 & 0 & 0 & \cdots & 0 & 0 \\
0 & 0 & 1 & -4 & 6 & -4 & 1 & 0 & 0 & 0 & \cdots & 0 & 0 \\
0 & 0 & 0 & 1 & -4 & 6 & -4 & 1 & 0 & 0 & \cdots & 0 & 0 \\
0 & 0 & 0 & 0 & 1 & 0 & 0 & 0 & 0 & 0 & \cdots & 0 & 0
\end{array}\right),
$$

its first three rows and the fourth row are obtained from (18) and (2), respectively,

$$
\mathbb{S}_{R_{1}}=\left(\begin{array}{cccccccccccccc}
0 & 0 & \cdots & 0 & 0 & 0 & 0 & 0 & 0 & 1 & 0 & 0 & 0 & 0 \\
0 & 0 & \cdots & 0 & 0 & 0 & 1 & -4 & 6 & -4 & 1 & 0 & 0 & 0 \\
0 & 0 & \cdots & 0 & 0 & 0 & 0 & 1 & -4 & 6 & -4 & 1 & 0 & 0 \\
0 & 0 & \cdots & 0 & 0 & 0 & 0 & 0 & 1 & -4 & 6 & -4 & 1 & 0
\end{array}\right),
$$

its first row and the last three rows are obtained from (2) and (19), respectively,

$$
\mathbb{G}=\left(0,0,0, \alpha_{0}, \mathbb{G}_{1}^{T}, \alpha_{1}, 0,0,0\right)^{T},
$$

while the matrices $\mathbb{D}$ and $\mathbb{G}_{1}$ are defined in (16) and (17), respectively.

By C-2 constraints: If we use (2), (12), (20) and (21) then the system can be expressed as

$$
\mathbb{S}_{2} \mathbb{D}=\mathbb{G}
$$

where $\mathbb{S}_{2}=\left(\mathbb{S}_{L_{2}}^{T}, \mathbb{S}^{T}, \mathbb{S}_{R_{2}}^{T}\right)^{T}$ while the first three rows and the last row of $\left[\mathbb{S}_{L_{2}}\right]_{4 \times(m+9)}$ are obtained from (20) and (2), respectively. Similarly the first row and the remaining three rows of $\left[\mathbb{S}_{R_{2}}\right]_{4 \times(m+9)}$ are obtained from (2) and (21), respectively. Now we have two systems, i.e., (22) and (24).

\subsection{Existence of the solution}

The matrices $\mathbb{S}_{1}$ and $\mathbb{S}_{2}$ involved in the systems (22) and (24) are non-singular. Their nonsingularity can be checked by finding their eigenvalues. We notice that for $m \leq 500$ the eigenvalues are nonzero. By [16], these are non-singular. Their singularity is not guaranteed for $m>500$. 


\section{Error estimation of the algorithm}

This section discussed the mathematical results as regards the convergence of the proposed method.

Let the analytic solution of the SPBVPs problem (1) with (2) be denoted as $Z_{e}$ then

$$
\varepsilon Z_{e}^{\bullet \bullet}(v)=p(v) Z_{e}^{\bullet}(v)+q(v) Z_{e}(v)+g(v) .
$$

It implies for node points, $\kappa=0,1, \ldots, m$,

$$
a y_{e}^{\bullet \bullet}\left(v_{\kappa}\right)=p\left(v_{\kappa}\right) Z_{e}^{\bullet}\left(v_{\kappa}\right)+q\left(v_{\kappa}\right) Z_{e}\left(v_{\kappa}\right)+g\left(v_{\kappa}\right) .
$$

Let the vector $Z_{e}(v)$ be defined as

$$
Z_{e}(v)=\left(Z_{e}\left(v_{0}\right), Z_{e}\left(v_{1}\right), \ldots, Z_{e}\left(v_{m}\right)\right)^{T} .
$$

By Taylor's series

$$
\begin{aligned}
Z_{e}^{\bullet}\left(v_{\kappa}\right)= & \frac{1}{25,878 h}\left[-256 Z_{e}\left(v_{\kappa}-4 h\right)-3200 Z_{e}\left(v_{\kappa}-3 h\right)+19,673 Z_{e}\left(v_{\kappa}-2 h\right)\right. \\
& -54,600 Z_{e}\left(v_{\kappa}-h\right)+54,600 Z_{e}\left(v_{\kappa}+h\right)-19,673 Z_{e}\left(v_{\kappa}+2 h\right) \\
& \left.+3200 Z_{e}\left(v_{\kappa}+3 h\right)+256 Z_{e}\left(v_{\kappa}+4 h\right)\right]+\mathcal{O}\left(h^{4}\right)
\end{aligned}
$$

and

$$
\begin{aligned}
Z_{e}^{\bullet \bullet}\left(v_{\kappa}\right)= & \frac{1}{8448 h^{2}}\left[256 Z_{e}\left(v_{\kappa}-4 h\right)+1600 Z_{e}\left(v_{\kappa}-3 h\right)-4725 Z_{e}\left(v_{\kappa}-2 h\right)\right. \\
& +17,300 Z_{e}\left(v_{\kappa}-h\right)-28,862 Z_{e}\left(v_{\kappa}\right)+17,300 Z_{e}\left(v_{\kappa}+h\right)-4725 Z_{e}\left(v_{\kappa}+2 h\right) \\
& \left.+1600 Z_{e}\left(v_{\kappa}+3 h\right)+256 Z_{e}\left(v_{\kappa}+4 h\right)\right]+\mathcal{O}\left(h^{4}\right) .
\end{aligned}
$$

Since $D(v)$ is the approximate solution of (1) which can be obtained from the system (22) or (24), by (7), for $\kappa=0,1, \ldots, m$, we have

$$
\varepsilon D^{\bullet \bullet}\left(v_{\kappa}\right)=p\left(v_{\kappa}\right) D^{\bullet}\left(v_{\kappa}\right)+q\left(v_{\kappa}\right) D\left(v_{\kappa}\right)+g\left(v_{\kappa}\right),
$$

where $D^{\bullet}\left(v_{\kappa}\right)$ and $D^{\bullet \bullet}\left(v_{\kappa}\right)$ are defined as

$$
\begin{aligned}
D^{\bullet}\left(v_{\kappa}\right)= & \frac{1}{25,878 h}\left[-256 d\left(v_{\kappa}-4 h\right)-3200 d\left(v_{\kappa}-3 h\right)+19,673 d\left(v_{\kappa}-2 h\right)\right. \\
& -54,600 d\left(v_{\kappa}-h\right)+54,600 d\left(v_{\kappa}+h\right)-19,673 d\left(v_{\kappa}+2 h\right) \\
& \left.+3200 d\left(v_{\kappa}+3 h\right)+256 d\left(v_{\kappa}+4 h\right)\right]+\mathcal{O}\left(h^{4}\right)
\end{aligned}
$$

and

$$
\begin{aligned}
D^{\bullet \bullet}\left(v_{\kappa}\right)= & \frac{1}{8448 h^{2}}\left[256 d\left(v_{\kappa}-4 h\right)+1600 d\left(v_{\kappa}-3 h\right)-4725 d\left(v_{\kappa}-2 h\right)\right. \\
& +17,300 d\left(v_{\kappa}-h\right)-28,862 d\left(v_{\kappa}\right)+17,300 d\left(v_{\kappa}+h\right)-4725 d\left(v_{\kappa}+2 h\right) \\
& \left.+1600 d\left(v_{\kappa}+3 h\right)+256 d\left(v_{\kappa}+4 h\right)\right]+\mathcal{O}\left(h^{4}\right) .
\end{aligned}
$$


Let the error function $\Delta(v)=Z_{e}(v)-D(v)$ and

$$
\Delta=\left(\Delta_{-4}, \Delta_{-3}, \ldots, \Delta_{m+3}, \Delta_{m+4}\right) .
$$

Then error vector at the given nodal values is

$$
\Delta\left(v_{\kappa}\right)=Z_{e}\left(v_{\kappa}\right)-D\left(v_{\kappa}\right), \quad-4 \leq \kappa \leq m+4 .
$$

This implies

$$
\begin{aligned}
& \Delta^{\bullet}\left(v_{\kappa}\right)=Z_{e}^{\bullet}\left(v_{\kappa}\right)-D^{\bullet}\left(v_{\kappa}\right), \quad-4 \leq \kappa \leq m+4, \\
& \Delta^{\bullet \bullet}\left(v_{\kappa}\right)=Z_{e}^{\bullet \bullet}\left(v_{\kappa}\right)-D^{\bullet \bullet}\left(v_{\kappa}\right), \quad-4 \leq \kappa \leq m+4 .
\end{aligned}
$$

The following result is obtained after subtracting (26) from (25):

$$
\varepsilon\left[Z_{e}^{\bullet \bullet}\left(v_{\kappa}\right)-D^{\bullet \bullet}\left(v_{\kappa}\right)\right]=p\left(v_{\kappa}\right)\left[Z_{e}^{\bullet}\left(v_{\kappa}\right)-D^{\bullet}\left(v_{\kappa}\right)\right]+q\left(v_{\kappa}\right)\left[Z_{e}\left(v_{\kappa}\right)-D\left(v_{\kappa}\right)\right] .
$$

By applying the definition of error vector the above equation can be written as

$$
\varepsilon \Delta^{\bullet \bullet}\left(v_{\kappa}\right)=p\left(v_{\kappa}\right) \Delta^{\bullet}\left(v_{\kappa}\right)+q\left(v_{\kappa}\right) \Delta\left(v_{\kappa}\right), \quad 0 \leq \kappa \leq m .
$$

This implies

$$
\varepsilon \Delta^{\bullet \bullet}\left(v_{\kappa}\right)-p\left(v_{\kappa}\right) \Delta^{\bullet}\left(v_{\kappa}\right)-q\left(v_{\kappa}\right) \Delta\left(v_{\kappa}\right)=0, \quad 0 \leq \kappa \leq m,
$$

where for $0 \leq \kappa \leq m$

$$
\begin{aligned}
\Delta^{\bullet}\left(v_{\kappa}\right)= & \frac{1}{25,878 h}\left[-256 \Delta\left(v_{\kappa}-4 h\right)-3200 \Delta\left(v_{\kappa}-3 h\right)+19,673 \Delta\left(v_{\kappa}-2 h\right)\right. \\
& -54,600 \Delta\left(v_{\kappa}-h\right)+54,600 \Delta\left(v_{\kappa}+h\right)-19,673 \Delta\left(v_{\kappa}+2 h\right) \\
& \left.+3200 \Delta\left(v_{\kappa}+3 h\right)+256 \Delta\left(v_{\kappa}+4 h\right)\right]+\mathcal{O}\left(h^{4}\right)
\end{aligned}
$$

and for $0 \leq \kappa \leq m$

$$
\begin{aligned}
\Delta \bullet \bullet\left(v_{\kappa}\right)= & \frac{1}{8448 h^{2}}\left[256 \Delta\left(v_{\kappa}-4 h\right)+1600 \Delta\left(v_{\kappa}-3 h\right)-4725 \Delta\left(v_{\kappa}-2 h\right)\right. \\
& +17,300 \Delta\left(v_{\kappa}-h\right)-28,862 \Delta\left(v_{\kappa}\right)+17,300 \Delta\left(v_{\kappa}+h\right)-4725 \Delta\left(v_{\kappa}+2 h\right) \\
& \left.+1600 \Delta\left(v_{\kappa}+3 h\right)+256 \Delta\left(v_{\kappa}+4 h\right)\right]+\mathcal{O}\left(h^{4}\right) .
\end{aligned}
$$

As $0 \leq v \leq 1$ and $v_{\kappa}=\kappa h, \kappa=0,1,2, \ldots, m$, the values lie outside the interval [0,1], i.e., $\Delta_{-4}, \ldots, \Delta_{-1}$ and $\Delta_{m+1}, \ldots, \Delta_{m+4}$ must be equal to zero. These error values can be assumed to be

$$
\Delta_{\kappa}= \begin{cases}\max _{0 \leq k \leq 4}\left\{\left|\Delta_{k}\right|\right\} \mathcal{O}\left(h^{4}\right), & -4 \leq \kappa<0, \\ \max _{m-4 \leq k \leq m}\left\{\left|\Delta_{k}\right|\right\} \mathcal{O}\left(h^{4}\right), & m<\kappa \leq m+4 .\end{cases}
$$


If we expand (27) by adopting a similar procedure to Proposition 1 then we obtain

$$
\left(\mathbb{S}_{1}+\mathcal{O}\left(h^{4}\right)-\mathcal{O}(h)\right) \Delta=0
$$

and

$$
\left(\mathbb{S}_{2}+\mathcal{O}\left(h^{4}\right)-\mathcal{O}(h)\right) \Delta=0 .
$$

Or equivalently

$$
\left(\mathbb{S}_{1}+\mathcal{O}\left(h^{4}\right)-\mathcal{O}(h)\right) \Delta=\mathcal{O}\left(h^{4}\right)\|\Delta\|=\mathcal{O}\left(h^{4}\right)
$$

and

$$
\left(\mathbb{S}_{2}+\mathcal{O}\left(h^{4}\right)\right) \Delta=\mathcal{O}\left(h^{4}\right)\|\Delta\|=\mathcal{O}\left(h^{4}\right) .
$$

The matrix $\mathbb{S}_{\kappa_{1}}+\mathcal{O}\left(h^{4}\right), \kappa_{1}=1,2$, for small $h$ and $\epsilon=0.1 \times 10^{-3}$, is non-singular so

$$
\|\Delta\| \leq\left(\left\|\mathbb{S}_{\kappa_{1}}^{-1}\right\|(1-\mathcal{O}(h))^{-1}\right) \mathcal{O}\left(h^{4}\right)=\mathcal{O}\left(h^{4}\right), \quad \kappa_{1}=1,2
$$

Hence $\|\Delta\|=O\left(h^{4}\right)$. This discussion can be summarized.

Proposition 2 Let $Z_{e}$ and $D_{\kappa}, \kappa=0,1, \ldots, m$ be the analytic and approximate solutions of second order SPBVPs defined in (1), respectively, then $\|\Delta\|=\left\|Z_{e}(v)-D(v)\right\| \leq \mathcal{O}\left(h^{4}\right)$.

Remark The order of error approximation varies if we use different values of $\epsilon$.

\section{Solutions of second order SPBVPs and discussions}

In this section, we consider second order SPBVPs and find their numerical solutions by using different algorithms. Since we have developed two linear systems i.e. (22) and (24) for approximate solutions of the SPBVPs, both systems have been used for solutions. We also give a comparison of solutions by computing the maximum absolute errors of the analytic and approximate solutions.

Example 4.1 This type of problem has also solved by [2, 3, 5, 6, 14],

$$
\varepsilon Z^{\bullet \bullet}(v)=Z+\cos ^{2}(\pi v)+2 \varepsilon \pi^{2} \cos (2 \pi v), \quad 0<v<1,
$$

where the boundary conditions of the above problem are

$$
Z(0)=0=Z(1)
$$

its analytic solution is

$$
Z(v)=\frac{\left[\operatorname{Exp}\left(\frac{-(1-v)}{\sqrt{\varepsilon}}\right)+\operatorname{Exp}\left(\frac{-v}{\sqrt{\varepsilon}}\right)\right]}{\left[1+\operatorname{Exp}\left(\frac{-1}{\sqrt{\varepsilon}}\right)\right]}-\cos ^{2}(\pi v) .
$$


Table 2 Maximum absolute errors (MAE) for Example 4.1

\begin{tabular}{lll}
\hline$m=10$ for $\varepsilon$ & Our C-1 & Our C-2 \\
\hline $0.1 \times 10^{-3}$ & $2.3445 \mathrm{E}-02$ & $1.8459 \mathrm{E}-02$ \\
$0.1 \times 10^{-4}$ & $2.4427 \mathrm{E}-03$ & $1.9197 \mathrm{E}-03$ \\
$0.1 \times 10^{-5}$ & $2.4525 \mathrm{E}-04$ & $1.9270 \mathrm{E}-04$ \\
$0.1 \times 10^{-6}$ & $2.4535 \mathrm{E}-05$ & $1.9278 \mathrm{E}-05$ \\
$0.1 \times 10^{-7}$ & $2.4535 \mathrm{E}-06$ & $1.9279 \mathrm{E}-06$ \\
$0.1 \times 10^{-8}$ & $2.4536 \mathrm{E}-07$ & $1.9279 \mathrm{E}-07$ \\
$0.1 \times 10^{-9}$ & $2.4536 \mathrm{E}-08$ & $1.9279 \mathrm{E}-08$ \\
\hline
\end{tabular}

Table 3 MAE in the solution of SPBVP in Example 4.1

\begin{tabular}{rlllllll}
\hline$m$ & \multicolumn{1}{c}{$\mathrm{C}-1$} & & & $\mathrm{C}-2$ & & \\
\cline { 2 - 3 } & $\varepsilon=10^{-5}$ & $\varepsilon=10^{-8}$ & $\varepsilon=10^{-10}$ & & $\varepsilon=10^{-5}$ & $\varepsilon=10^{-8}$ & $\varepsilon=10^{-10}$ \\
\hline 10 & $2.4427 \mathrm{E}-03$ & $2.4535 \mathrm{E}-06$ & $2.4536 \mathrm{E}-08$ & & $1.9197 \mathrm{E}-03$ & $1.9279 \mathrm{E}-06$ & $1.9278 \mathrm{E}-08$ \\
100 & $1.2005 \mathrm{E}-01$ & $2.3115 \mathrm{E}-04$ & $2.3126 \mathrm{E}-06$ & & $9.3578 \mathrm{E}-02$ & $1.8887 \mathrm{E}-04$ & $1.8895 \mathrm{E}-06$ \\
150 & $1.5152 \mathrm{E}-01$ & $5.1955 \mathrm{E}-04$ & $5.2008 \mathrm{E}-06$ & & $1.1209 \mathrm{E}-01$ & $4.2458 \mathrm{E}-04$ & $4.2498 \mathrm{E}-06$ \\
200 & $1.5950 \mathrm{E}-01$ & $9.2276 \mathrm{E}-04$ & $9.2442 \mathrm{E}-06$ & & $1.1327 \mathrm{E}-01$ & $7.5413 \mathrm{E}-04$ & $7.5541 \mathrm{E}-06$ \\
250 & $1.5692 \mathrm{E}-01$ & $1.4402 \mathrm{E}-03$ & $1.4443 \mathrm{E}-05$ & & $1.0808 \mathrm{E}-01$ & $1.1771 \mathrm{E}-03$ & $1.1802 \mathrm{E}-05$ \\
\hline
\end{tabular}

Table 4 MAE in the solution of SPBVP in Example 4.1

\begin{tabular}{lllll}
\hline & $\begin{array}{l}\varepsilon=10^{-5} \\
m=10\end{array}$ & $\begin{array}{l}\varepsilon=10^{-5} \\
m=100\end{array}$ & $\begin{array}{l}\varepsilon=10^{-8} \\
m=200\end{array}$ & $\begin{array}{l}\varepsilon=10^{-10} \\
m=250\end{array}$ \\
\hline$\lambda_{1}$ and $\lambda_{2}$ by $[2,6]$ & $\ldots$ & & & \\
$1 / 18,4 / 9$ & $\ldots$ & $1.44463 \mathrm{E}-03$ & $6.22342 \mathrm{E}-02$ & $6.27380 \mathrm{E}-02$ \\
$1 / 14,3 / 7$ & $\ldots$ & $1.52823 \mathrm{E}-02$ & $8.33647 \mathrm{E}-02$ & $8.39115 \mathrm{E}-02$ \\
$1 / 24,11 / 24$ & $\ldots$ & $1.00616 \mathrm{E}-02$ & $4.50702 \mathrm{E}-02$ & $4.55413 \mathrm{E}-02$ \\
$1 / 30,14 / 30$ & $\ldots$ & $1.67078 \mathrm{E}-02$ & $3.52995 \mathrm{E}-02$ & $3.57527 \mathrm{E}-02$ \\
$1 / 6,1 / 3$ & $\ldots$ & $1.1971 \mathrm{E}-01$ & $2.6683 \mathrm{E}-01$ & $2.6793 \mathrm{E}-01$ \\
Our C-1 & $2.4427 \mathrm{E}-03$ & $1.2005 \mathrm{E}-01$ & $9.2276 \mathrm{E}-04$ & $1.4443 \mathrm{E}-05$ \\
Our C-2 & $1.9197 \mathrm{E}-03$ & $9.3579 \mathrm{E}-02$ & $7.5413 \mathrm{E}-04$ & $1.1802 \mathrm{E}-05$ \\
\hline
\end{tabular}

Table 5 MAE in the solution of SPBVP in Example 4.1

\begin{tabular}{lllll}
\hline For $m=32$ and $\varepsilon=\left(2^{-r}\right)^{2}$ & Bawa et al. [3] & Sapna et al. [14] & Our C-1 & Our C-2 \\
\hline$r=10$ & $5.022 \mathrm{E}-02$ & $1.23 \mathrm{E}-02$ & $2.2646 \mathrm{E}-03$ & $1.8478 \mathrm{E}-03$ \\
$r=20$ & $3.125 \mathrm{E}-02$ & $1.23 \mathrm{E}-08$ & $2.1692 \mathrm{E}-09$ & $1.7695 \mathrm{E}-09$ \\
$r=25$ & $3.125 \mathrm{E}-02$ & $1.20 \mathrm{E}-11$ & $2.1200 \mathrm{E}-12$ & $1.7283 \mathrm{E}-12$ \\
\hline
\end{tabular}

Table 6 MAE in the solution of SPBVP in Example 4.1

\begin{tabular}{lllll}
\hline For $m=32$ and $\varepsilon=2^{-r}$ & Kadalbajoo et al. [5] & Sapna et al. & Our C-1 & Our C-2 \\
\hline$r=10$ & $5.022 \mathrm{E}-02$ & $1.80 \mathrm{E}-03$ & $1.5551 \mathrm{E}-01$ & $1.0545 \mathrm{E}-01$ \\
$r=20$ & $3.125 \mathrm{E}-02$ & $1.23 \mathrm{E}-03$ & $2.2645 \mathrm{E}-03$ & $1.8478 \mathrm{E}-03$ \\
$r=25$ & $3.125 \mathrm{E}-02$ & $4.04 \mathrm{E}-4$ & $7.1071 \mathrm{E}-05$ & $5.7977 \mathrm{E}-05$ \\
\hline
\end{tabular}

Example 4.2 Consider the boundary value problem [10, 12, 13]

$$
\varepsilon Z^{\bullet \bullet}(v)-(1+v) Z(v)=40\left[v\left(v^{2}-1\right)-2 \varepsilon\right], \quad 0<v<1,
$$






(a)

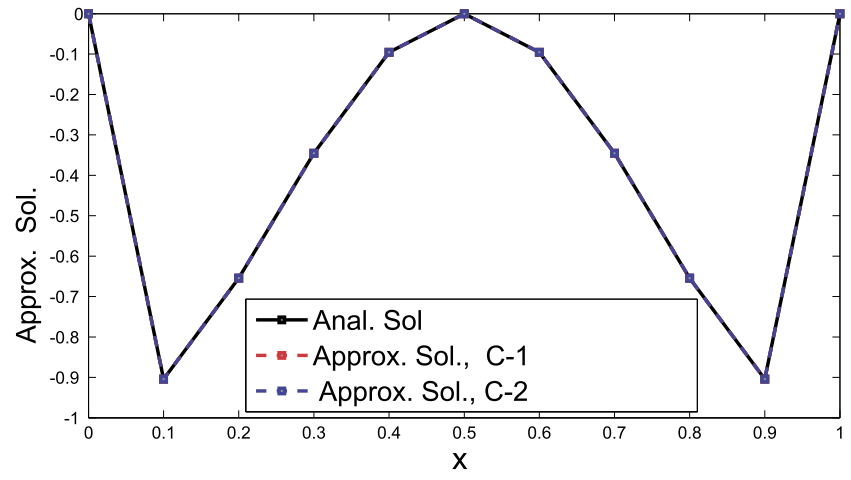

(b)

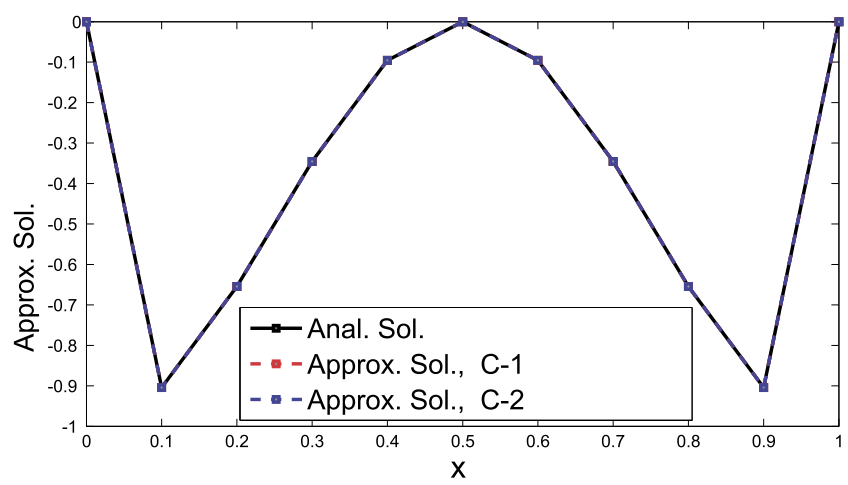

(c)

Figure 1 Comparison concerning Example 4.1: Analytic and approximate solutions with parametric setting: $N=10$ and $\varepsilon=10^{-4}, 10^{-7}, 10^{-10}$

where the boundary conditions of the above problem are

$$
Z(0)=0=Z(1),
$$

its analytic solution is

$$
Z(v)=40 v(1-v) .
$$


Example 4.3 Take the problem already solved by [7, 9],

$$
\begin{aligned}
\varepsilon Z^{\bullet \bullet}(v)-\{1+v(1-v)\} Z(v)= & -\left[1+v(1-v)+\left\{2 \sqrt{\varepsilon}-v^{2}(1-v)\right\}\right. \\
& \times e^{\left\{\frac{(1-v)}{\sqrt{\varepsilon}}\right\}}+\left\{2 \sqrt{\varepsilon}-v(1-v)^{2}\right\} e^{\left\{-\frac{v}{\sqrt{\varepsilon}}\right\}},
\end{aligned}
$$

here $0 \leq v \leq 1$ and

$$
Z(0)=Z(1)=0,
$$

its analytic solution is

$$
Z(v)=1+(v-1) e^{\left\{-\frac{v}{\sqrt{\varepsilon}}\right\}}-x e^{\left\{-\frac{(1-v)}{\sqrt{\varepsilon}}\right\}} .
$$

\subsection{Discussion and comparison}

We solve SPBVPs by our algorithm and summarized the results in the following form.

- The facts regarding the solutions of Example 4.1 are shown in Tables 2-6 and in Figs. 1-3 In Tables 2 and 3, the maximum absolute errors (MAE) are given while in Tables 4-6 the comparison with the methods of [2, 3, 5, 6, 14] are presented. In Fig. 1 the solutions are presented. In Figs. 2 and 3 results for $m$ and $\varepsilon$ are depicted.

- In Tables 7-10 and in Figs. 4-6 results of Example 4.2 are presented. Tables 7 and 10 show the MAE while Tables 9 and 10 present the comparison of MAE with $[10,12,13]$. This shows our results are better. Figure 4 shows the solutions while Figs. 5 and 6 show the results for $m$ and $\varepsilon$.

- Tables 11-13 and Figs. 7-9 are related to the solution of Example 4.3. The MAE are shown in Tables 11 and 12. We compare our result with the results of [7,9] and found them to be better. The graphical representation is given in Figs. 8 and 9

- From these results we conclude that the condition C-2 gives better results than the condition C-1.

- If we keep $m$ fixed, then MAE increases with the increase of $\varepsilon$. It is also observed that if we keep $\varepsilon$ fixed, then MAE decreases with the increase of $m$.

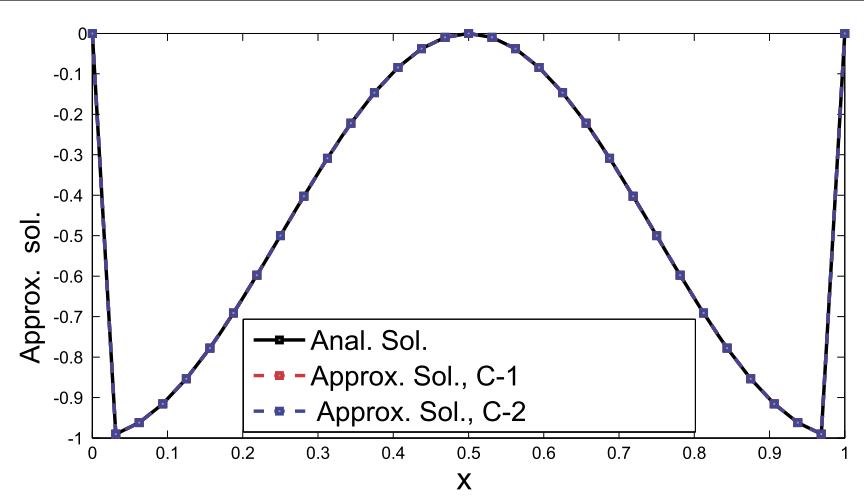

Figure 2 Comparison concerning Example 4.1: Analytic and approximate solutions with parametric setting: $N=32$ and $\varepsilon=2^{-25}$ 


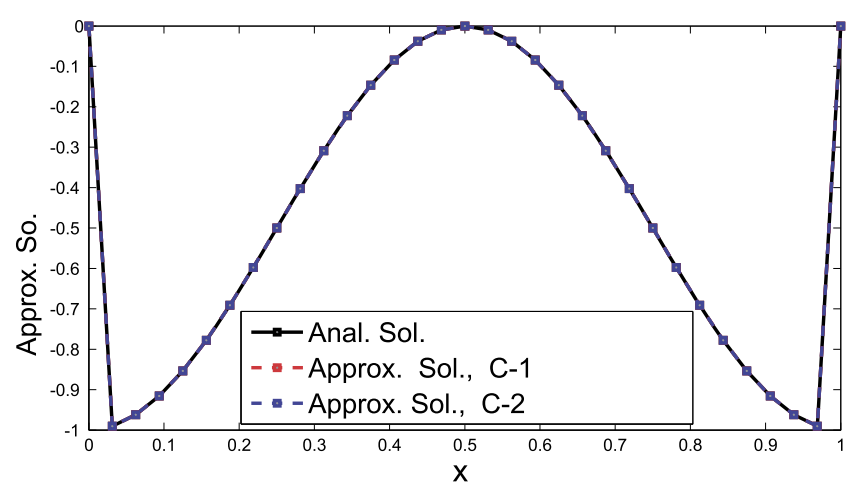

Figure 3 Comparison concerning Example 4.1: Analytic and approximate solutions with parametric setting: $N=32$ and $\varepsilon=\left(2^{-20}\right)^{2}$

Table 7 MAE in the solution of SPBVP in Example 4.2

\begin{tabular}{lll}
\hline$m=10$ for $\varepsilon$ & Our C-1 & Our C-2 \\
\hline $0.1 \times 10^{-3}$ & $1.4049 \mathrm{E}-02$ & $2.4080 \mathrm{E}-02$ \\
$0.1 \times 10^{-4}$ & $1.4331 \mathrm{E}-03$ & $2.4866 \mathrm{E}-03$ \\
$0.1 \times 10^{-5}$ & $1.4360 \mathrm{E}-04$ & $2.4948 \mathrm{E}-04$ \\
$0.1 \times 10^{-6}$ & $1.4363 \mathrm{E}-05$ & $2.4957 \mathrm{E}-05$ \\
$0.1 \times 10^{-7}$ & $1.4364 \mathrm{E}-06$ & $2.4958 \mathrm{E}-06$ \\
$0.1 \times 10^{-8}$ & $1.4364 \mathrm{E}-07$ & $2.4958 \mathrm{E}-07$ \\
$0.1 \times 10^{-9}$ & $1.4364 \mathrm{E}-08$ & $2.4958 \mathrm{E}-08$ \\
\hline
\end{tabular}

Table 8 MAE in the solution of SPBVP in Example 4.2

\begin{tabular}{|c|c|c|c|c|c|c|}
\hline \multirow[t]{2}{*}{$m$} & \multicolumn{3}{|l|}{$C-1$} & \multicolumn{3}{|l|}{$C-2$} \\
\hline & $\varepsilon=10^{-5}$ & $\varepsilon=10^{-8}$ & $\varepsilon=10^{-10}$ & $\varepsilon=10^{-5}$ & $\varepsilon=10^{-8}$ & $\varepsilon=10^{-10}$ \\
\hline 10 & $1.4331 E-03$ & $1.4364 \mathrm{E}-06$ & $1.4364 \mathrm{E}-08$ & $2.4866 \mathrm{E}-03$ & $2.4958 \mathrm{E}-06$ & $2.4958 \mathrm{E}-08$ \\
\hline 100 & $1.5099 \mathrm{E}-03$ & $1.5640 \mathrm{E}-06$ & $1.5644 \mathrm{E}-08$ & $1.1720 \mathrm{E}-02$ & $1.7289 \mathrm{E}-05$ & $1.7298 \mathrm{E}-07$ \\
\hline 150 & $1.5217 \mathrm{E}-03$ & $1.5687 \mathrm{E}-06$ & $1.5695 \mathrm{E}-08$ & 1.3907E-02 & $2.6851 \mathrm{E}-05$ & $2.6881 E-07$ \\
\hline 200 & $1.5262 \mathrm{E}-03$ & $1.5706 \mathrm{E}-06$ & $1.5721 \mathrm{E}-08$ & $1.4855 \mathrm{E}-02$ & $3.6398 \mathrm{E}-05$ & $3.6471 \mathrm{E}-07$ \\
\hline 250 & $1.528 \mathrm{E}-03$ & $1.5714 \mathrm{E}-06$ & $1.5736 \mathrm{E}-08$ & $1.5259 \mathrm{E}-02$ & $4.5919 \mathrm{E}-05$ & $4.6062 \mathrm{E}-07$ \\
\hline
\end{tabular}

Table 9 MAE in the solution of SPBVP in Example 4.2

\begin{tabular}{llllll}
\hline For $m=16$ and $\varepsilon$ & Method in [10] & Method in [12] & Method in [13] & Our C-1 & Our C-2 \\
\hline $0.1 \times 10^{-3}$ & $0.25 \mathrm{E}-01$ & $0.26 \mathrm{E}-01$ & $0.65 \mathrm{E}-04$ & $0.1408 \mathrm{E}-01$ & $0.3079 \mathrm{E}-01$ \\
$0.1 \times 10^{-4}$ & $0.21 \mathrm{E}-01$ & $0.24 \mathrm{E}-01$ & $0.36 \mathrm{E}-04$ & $0.1478 \mathrm{E}-02$ & $0.3374 \mathrm{E}-02$ \\
$0.1 \times 10^{-5}$ & $0.70 \mathrm{E}-02$ & $0.17 \mathrm{E}-01$ & $0.33 \mathrm{E}-04$ & $0.14862 \mathrm{E}-03$ & $0.3407 \mathrm{E}-03$ \\
$0.1 \times 10^{-6}$ & $0.75 \mathrm{E}-03$ & $0.69 \mathrm{E}-02$ & $0.26 \mathrm{E}-04$ & $0.14870 \mathrm{E}-04$ & $0.3410 \mathrm{E}-04$ \\
$0.1 \times 10^{-7}$ & $0.74 \mathrm{E}-04$ & $0.23 \mathrm{E}-02$ & $0.20 \mathrm{E}-04$ & $0.14871 \mathrm{E}-05$ & $0.3411 \mathrm{E}-05$ \\
$0.1 \times 10^{-8}$ & $0.67 \mathrm{E}-05$ & $0.76 \mathrm{E}-03$ & $0.11 \mathrm{E}-04$ & $0.14872 \mathrm{E}-06$ & $0.3411 \mathrm{E}-06$ \\
\hline
\end{tabular}

Table 10 MAE in the solution of SPBVP in Example 4.2

\begin{tabular}{llllll}
\hline For $m=32$ and $\varepsilon$ & Method in [10] & Method in [12] & Method in [13] & Our C-1 & Our C-2 \\
\hline $0.1 \times 10^{-3}$ & $0.64 \mathrm{E}-02$ & $0.65 \mathrm{E}-02$ & $0.59 \mathrm{E}-04$ & $0.1414 \mathrm{E}-01$ & $0.3861 \mathrm{E}-01$ \\
$0.1 \times 10^{-4}$ & $0.61 \mathrm{E}-02$ & $0.64 \mathrm{E}-02$ & $0.21 \mathrm{E}-04$ & $0.1497 \mathrm{E}-02$ & $0.5501 \mathrm{E}-02$ \\
$0.1 \times 10^{-5}$ & $0.41 \mathrm{E}-02$ & $0.56 \mathrm{E}-02$ & $0.35 \mathrm{E}-04$ & $0.1529 \mathrm{E}-03$ & $0.5756 \mathrm{E}-03$ \\
$0.1 \times 10^{-6}$ & $0.77 \mathrm{E}-03$ & $0.31 \mathrm{E}-02$ & $0.39 \mathrm{E}-04$ & $0.1531 \mathrm{E}-04$ & $0.5783 \mathrm{E}-04$ \\
$0.1 \times 10^{-7}$ & $0.76 \mathrm{E}-04$ & $0.12 \mathrm{E}-02$ & $0.21 \mathrm{E}-04$ & $0.1532 \mathrm{E}-05$ & $0.5786 \mathrm{E}-05$ \\
$0.1 \times 10^{-8}$ & $0.67 \mathrm{E}-05$ & $0.38 \mathrm{E}-03$ & $0.21 \mathrm{E}-04$ & $0.1532 \mathrm{E}-06$ & $0.5786 \mathrm{E}-06$ \\
\hline
\end{tabular}




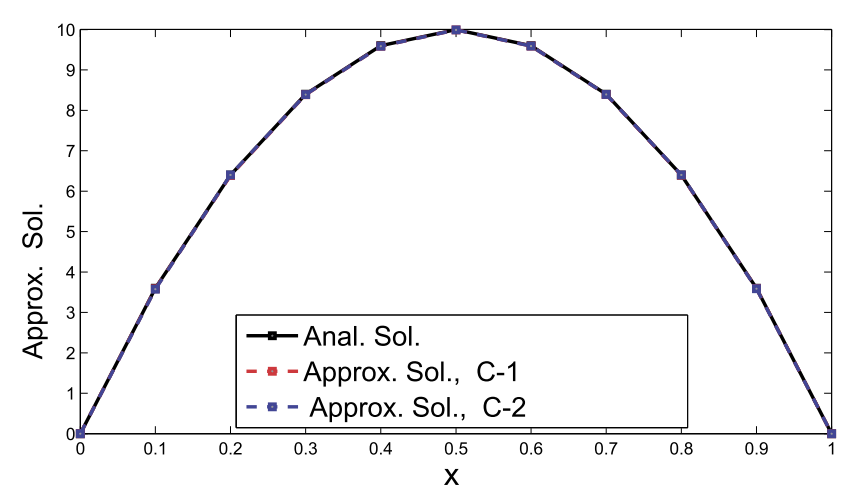

(a)

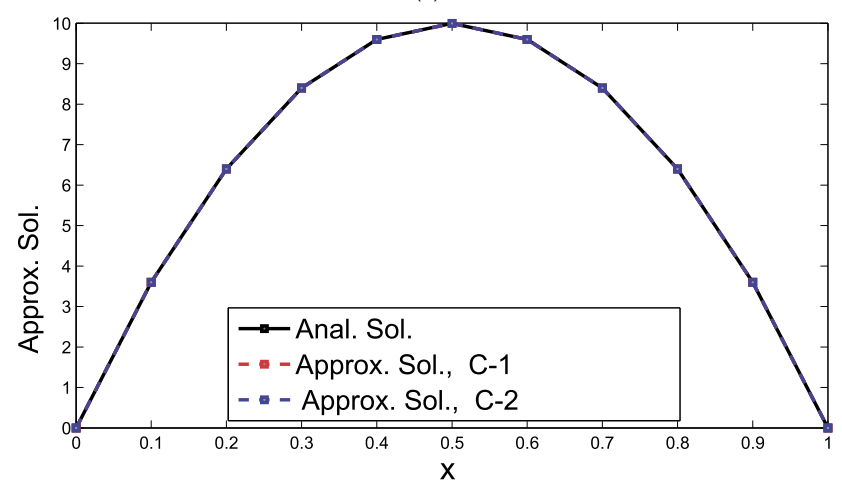

(b)

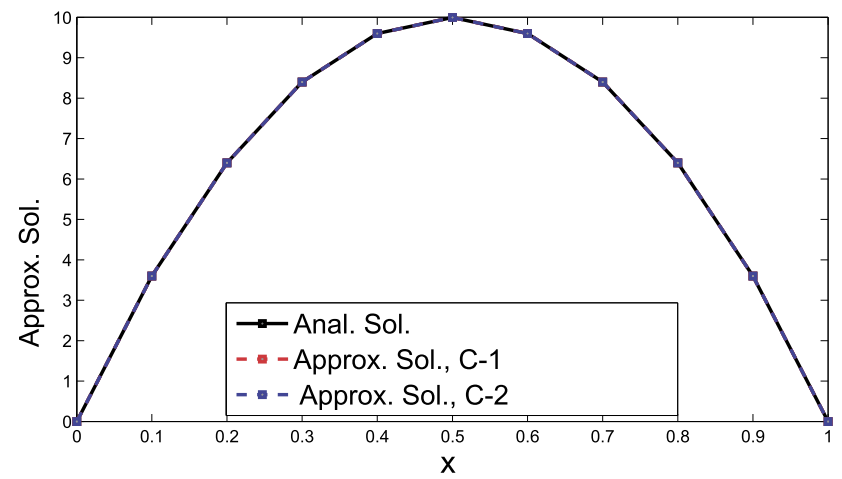

(c)

Figure 4 Comparison concerning Example 4.2: Analytic and approximate solutions with parametric setting: $N=10$ with $\varepsilon=10^{-4}, 10^{-7}, 10^{-10}$

\section{Conclusions}

In this paper, we introduced a numerical algorithm for the solution of second order SPBVPS. The algorithm was developed by using the 2-scale relation of a well-known interpolating subdivision scheme. This algorithm gives the approximate solution of second order SPBVPS with a fourth order of approximation. We presented the comparison of maximum absolute error of the solutions obtained from subdivision (i.e. our method), spline $[2,3,5,6]$, finite difference $[9,10,12,13]$ and Haar wavelet $[7,14]$ algorithms. We concluded that our algorithm gives smaller maximum absolute error. 


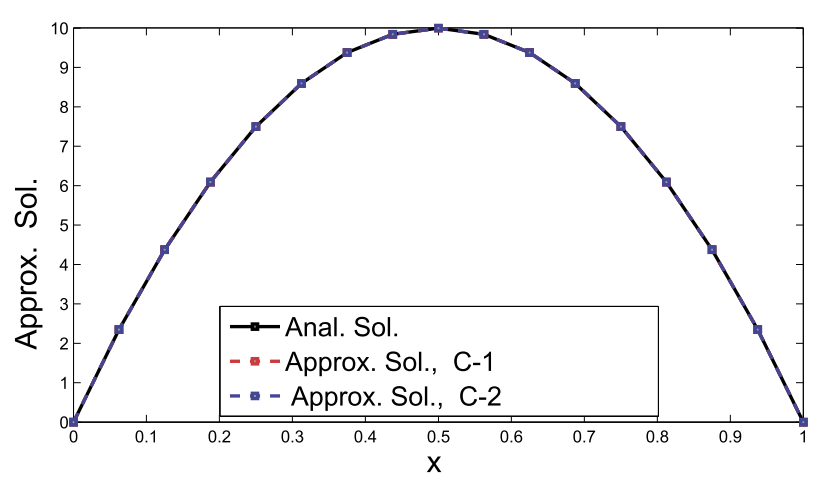

Figure 5 Comparison concerning Example 4.2: Analytic and approximate solutions with parametric setting: $N=16$ and $\varepsilon=10^{-8}$



Figure 6 Comparison concerning Example 4.2: Analytic and approximate solutions with parametric setting: $N=32$ and $\varepsilon=10^{-9}$

Table 11 MAE in the solution of SPBVP in Example 4.3

\begin{tabular}{lll}
\hline$m=10$ for $\varepsilon$ & Our C-1 & Our C-2 \\
\hline $0.1 \times 10^{-3}$ & $2.0312 \mathrm{E}-02$ & $7.4455 \mathrm{E}-03$ \\
$0.1 \times 10^{-4}$ & $2.1110 \mathrm{E}-03$ & $7.6536 \mathrm{E}-04$ \\
$0.1 \times 10^{-5}$ & $2.1189 \mathrm{E}-04$ & $7.6679 \mathrm{E}-05$ \\
$0.1 \times 10^{-6}$ & $2.1197 \mathrm{E}-05$ & $7.6682 \mathrm{E}-06$ \\
$0.1 \times 10^{-7}$ & $2.1198 \mathrm{E}-06$ & $7.6679 \mathrm{E}-07$ \\
$0.1 \times 10^{-8}$ & $2.1198 \mathrm{E}-07$ & $7.6678 \mathrm{E}-08$ \\
$0.1 \times 10^{-9}$ & $2.1198 \mathrm{E}-08$ & $7.6679 \mathrm{E}-09$ \\
\hline
\end{tabular}

Table 12 MAE in the solution of SPBVP in Example 4.3

\begin{tabular}{rlllllll}
\hline$m$ & $\mathrm{C}-1$ & & & & $\mathrm{C}-2$ & & \\
& $\varepsilon=10^{-5}$ & $\varepsilon=10^{-8}$ & $\varepsilon=10^{-10}$ & & $\varepsilon=10^{-5}$ & $\varepsilon=10^{-8}$ & $\varepsilon=10^{-10}$ \\
\hline 10 & $2.1110 \mathrm{E}-03$ & $2.1198 \mathrm{E}-06$ & $2.1198 \mathrm{E}-08$ & & $7.6536 \mathrm{E}-04$ & $7.6679 \mathrm{E}-07$ & $7.6677 \mathrm{E}-09$ \\
16 & $5.5268 \mathrm{E}-03$ & $5.5876 \mathrm{E}-06$ & $5.5877 \mathrm{E}-08$ & & $2.0082 \mathrm{E}-03$ & $2.0212 \mathrm{E}-06$ & $2.0212 \mathrm{E}-08$ \\
32 & $2.1930 \mathrm{E}-02$ & $2.2964 \mathrm{E}-05$ & $2.2965 \mathrm{E}-07$ & & $8.0232 \mathrm{E}-03$ & $8.3069 \mathrm{E}-06$ & $8.3069 \mathrm{E}-08$ \\
100 & $1.1919 \mathrm{E}-01$ & $2.2869 \mathrm{E}-04$ & $2.2879 \mathrm{E}-06$ & & $2.3666 \mathrm{E}-02$ & $8.2735 \mathrm{E}-05$ & $8.2759 \mathrm{E}-07$ \\
200 & $1.5931 \mathrm{E}-01$ & $9.1801 \mathrm{E}-04$ & $9.1965 \mathrm{E}-06$ & & $3.7244 \mathrm{E}-02$ & $3.3226 \mathrm{E}-04$ & $3.3265 \mathrm{E}-06$ \\
250 & $1.5691 \mathrm{E}-01$ & $1.4343 \mathrm{E}-03$ & $1.4384 \mathrm{E}-05$ & & $4.6497 \mathrm{E}-02$ & $5.1930 \mathrm{E}-04$ & $5.2028 \mathrm{E}-06$ \\
\hline
\end{tabular}


Table 13 MAE in the solution of SPBVP in Example 4.3

\begin{tabular}{|c|c|c|c|c|}
\hline For $m=16$ and $\varepsilon=10^{-r}$ & Kumar [7] & Lubuma [9] & Our C-1 & Our C-2 \\
\hline$r=3$ & $0.77 \mathrm{E}-01$ & $0.28 \mathrm{E}-01$ & $0.1524 E-00$ & $0.2703 E-01$ \\
\hline$r=5$ & $0.46 \mathrm{E}-02$ & $0.53 E-02$ & $0.5527 \mathrm{E}-02$ & $0.2008 \mathrm{E}-02$ \\
\hline$r=7$ & $0.46 \mathrm{E}-04$ & $0.53 \mathrm{E}-02$ & $0.5587 \mathrm{E}-04$ & $0.2021 \mathrm{E}-04$ \\
\hline$r=8$ & $0.46 \mathrm{E}-05$ & $0.53 E-02$ & $0.5589 E-05$ & $2.0212 \mathrm{E}-05$ \\
\hline
\end{tabular}

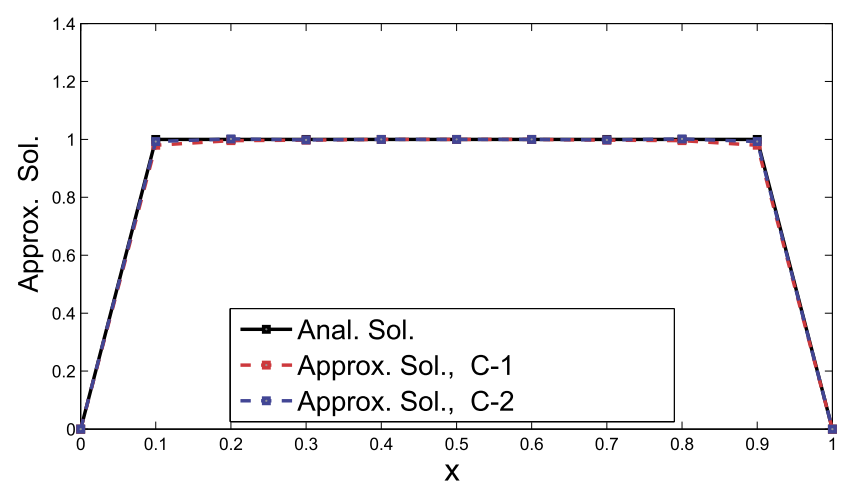

(a)

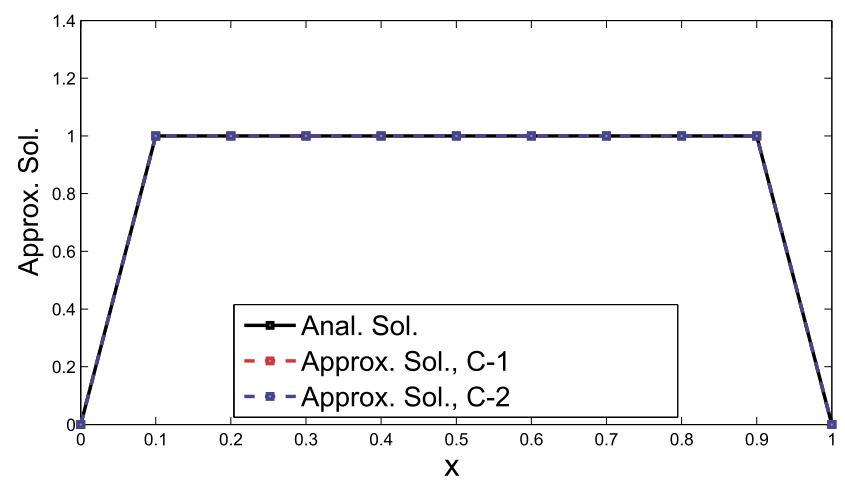

(b)

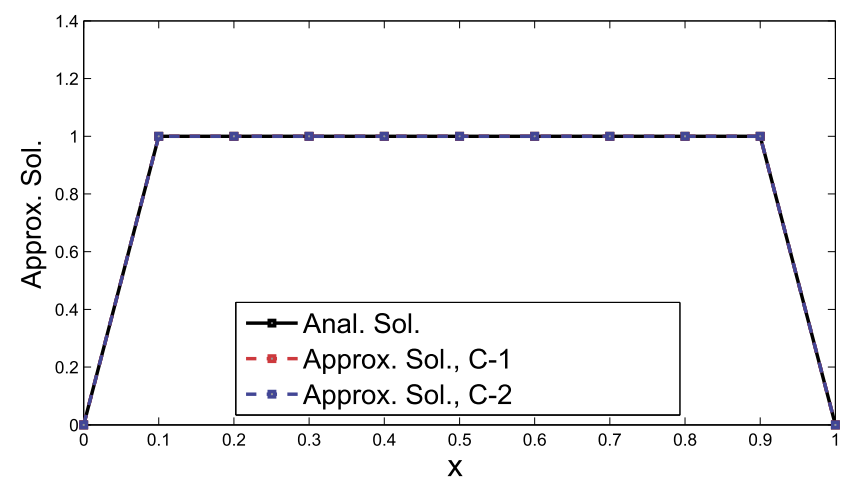

(c)

Figure 7 Comparison concerning Example 4.3: Analytic and approximate solutions with parametric setting: $N=10$ with $\varepsilon=10^{-4}, 10^{-7}, 10^{-10}$ shown in $(\mathbf{a}),(\mathbf{b})$ and $(\mathbf{c})$, respectively 


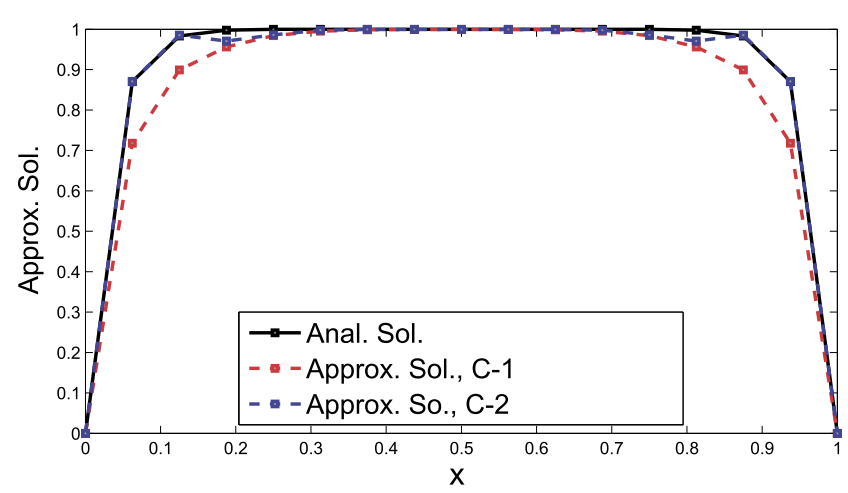

Figure 8 Comparison concerning Example 4.3: Analytic and approximate solutions with parametric setting: $N=16$ and $\varepsilon=10^{-5}$

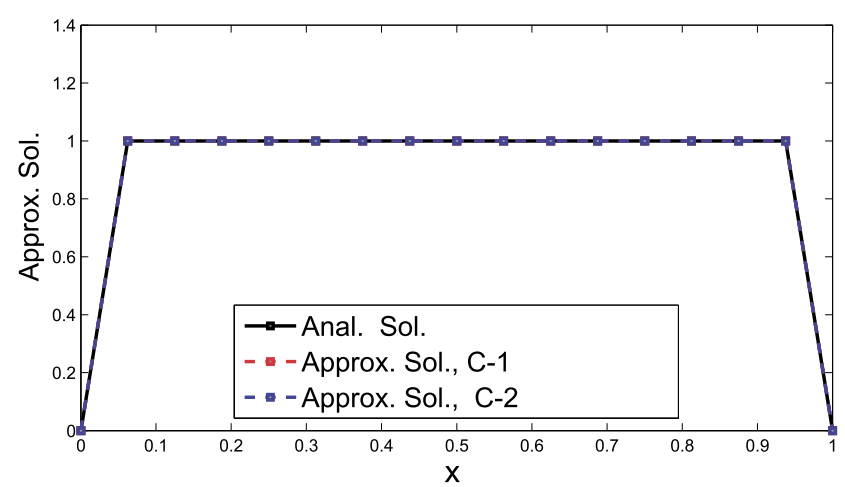

Figure 9 Comparison concerning Example 4.3: Analytic and approximate solutions with parametric setting: $N=16$ and $a=10^{-8}$

\section{Acknowledgements}

This work is supported by Indigenous Ph.D. Scholarship Scheme of HEC Pakistan. The first author is supported by HEC, NRPU (P. No. 3183) and second author is grateful to Higher Education Commission Pakistan for granting scholarship for Ph. D studies

\section{Funding}

"Not available."

\section{Availability of data and materials}

"Data sharing not applicable to this article as no datasets were generated or analysed during the current study."

\section{Competing interests}

The authors declare that they have no competing interests.

\section{Authors' contributions}

The authors have contributed equally to this manuscript. They read and approved the final manuscript.

\section{Author details}

'Department of Mathematics, The Islamia University of Bahawalpur, Bahawalpur, Pakistan. ${ }^{2}$ Department of Mathematics, The Government Sadiq College Women University Bahawalpur, Bahawalpur, Pakistan. ${ }^{3}$ Department of Mathematics, Cankaya University, Ankara, Turkey. ${ }^{4}$ Institute of Space Sciences, Magurele-Bucharest, Romania. ${ }^{5}$ China Medical University Hospital, China Medical University, Taichung, Taiwan. ${ }^{6}$ Informetrics Research Group, Ton Duc Thang University, Ho Chi Minh City, Vietnam. ${ }^{7}$ Faculty of Mathematics \& Statistics, Ton Duc Thang University, Ho Chi Minh City, Vietnam. ${ }^{8}$ Department of Mathematics, College of Arts and Sciences, Prince Sattam bin Abdulaziz University, Wadi Aldawaser, Saudi Arabia.

\section{Publisher's Note}

Springer Nature remains neutral with regard to jurisdictional claims in published maps and institutional affiliations. 
Received: 18 February 2020 Accepted: 27 May 2020 Published online: 11 June 2020

\section{References}

1. Ascher, U.M., Mattheij, R.M.M., Russell, R.D.: Numerical Solution of Boundary Value Problems for Ordinary Differential Equations. Prentice Hall, Englewood Cliffs (1988)

2. Aziz, T., Khan, A.: A spline method for second order singularly perturbed boundary value problems. J. Comput. Appl. Math. 147, 445-452 (2002)

3. Bawa, R.K., Natesan, S.: A computational method for self adjoint singular perturbation problem using quintic spline. Comput. Math. Appl. 50, 1371-1382 (2005)

4. Ejaz, S.T., Mustafa, G., Khan, F.: Subdivision schemes based collocation algorithms for solution of fourth order boundary value problems. Math. Probl. Eng. 2015, Article ID 240138 (2015)

5. Kadalbajoo, M.K., Aggarwal, V.K.: Fitted mesh B-spline collocation method for solving self-adjoint singularly perturbed boundary value problems. Appl. Math. Comput. 161, 973-987 (2005)

6. Khan, I., Aziz, T.: Tension spline method for second order singularly perturbed boundary value problems. Int. J. Comput. Math. 82(12), 1547-1553 (2005)

7. Kumar, V., Mehra, M.: Wavelet optimized finite difference method using interpolating wavelets for self-adjoint singularly perturbed problems. J. Comput. Appl. Math. 230(2), 803-812 (2009)

8. Lee, B.G., Lee, Y.J., Yoon, J.: Stationary binary subdivision schemes using radial basis function interpolation. Adv. Comput. Math. 25, 57-72 (2006)

9. Lubuma, J.M.S., Patidar, K.C.: Uniformly convergent non-standard finite difference methods for self-adjoint singularly perturbed problems. J. Comput. Appl. Math. 191, 228-238 (2006)

10. Miller, J.J.H.: On the convergence, uniformly in $\varepsilon$, of difference schemes for a two point singular perturbation problem. In: Numerical Analysis of Singular Perturbation Problems, pp. 467-474. Academic Press, New York (1979)

11. Mustafa, G., Ejaz, S.T.: Numerical solution of two point boundary value problems by interpolating subdivision schemes. Abstr. Appl. Anal. 2014, Article ID 721314 (2014)

12. Niijima, K.: On a finite difference scheme for a singular perturbation problem without a first derivative term I. Mem. Numer. Math. 7, 1-10 (1980)

13. Niijima, K.: On a finite difference scheme for a singular perturbation problem without a first derivative term II. Mem. Numer. Math. 7, 11-27 (1980)

14. Pandit, S., Kumar, M.: Haar wavelet approach for numerical solution of two parameters singularly perturbed boundary value problems. Appl. Math. Inf. Sci. 8(6), 2965-2974 (2014)

15. Qu, R., Agarwal, R.P.: Solving two point boundary value problems by interpolatory subdivision algorithms. Int. J. Comput. Math. 60, 279-294 (1996)

16. Strang, G.: Linear Algebra and Its Applications. Cengage Learning India Private Limited (2011). ISBN 81-315-0172-8

17. Tirmizi, I.A., i-Haq, F., ul-Islam, S.: Non-polynomial spline solution of singularly perturbed boundary value problems. Appl. Math. Comput. 196, 6-16 (2008)

\section{Submit your manuscript to a SpringerOpen ${ }^{\circ}$ journal and benefit from:}

- Convenient online submission

- Rigorous peer review

- Open access: articles freely available online

- High visibility within the field

- Retaining the copyright to your article

Submit your next manuscript at $\gg$ springeropen.com 